\title{
Morphometry of the Trabecular Meshwork In Vivo in a Healthy Population Using Fourier-Domain Optical Coherence Tomography
}

\author{
José I. Fernández-Vigo, ${ }^{1,2}$ Julián García-Feijóo, ${ }^{1}$ Jose M. Martínez-de-la-Casa, ${ }^{1}$ Javier García-Bella, ${ }^{1}$ \\ and José A. Fernández-Vigo ${ }^{2,3}$ \\ ${ }^{1}$ Clínico San Carlos University Hospital, Department of Ophthalmology, Institute of Health Research, Complutense University of \\ Madrid, Madrid, Spain \\ ${ }^{2}$ International Center of Advanced Ophthalmology, Madrid, Spain \\ ${ }^{3}$ Department of Ophthalmology, University of Extremadura, Badajoz, Spain
}

Correspondence: José I. FernándezVigo, c/Zurbano 71, 28010 Madrid, Spain;

jfvigo@hotmail.com.

Submitted: November 27, 2014

Accepted: February 11, 2015

Citation: Fernández-Vigo JI, GarcíaFeijóo J, Martínez-de-la-Casa JM, García-Bella J, Fernández-Vigo JA. Morphometry of the trabecular meshwork in vivo in a healthy population using Fourier-domain optical coherence tomography. Invest Ophthalmol Vis Sci. 2015;56:1782-1788.

DOI:10.1167/iovs.14-16154
Purpose. We measured the length, thickness, and area of the trabecular meshwork (TM) in vivo using Fourier domain optical coherence tomography (FD-OCT) in a Caucasian population of healthy subjects.

Methods. A cross-sectional study was done of 1006 healthy subjects. Left eyes were randomly selected. Age, sex, IOP, and spherical refractive error were noted. The depth and volume of the anterior chamber and the central corneal thickness were measured with Pentacam, while IOL Master was used to measure the axial length. The length, thickness, and area of the TM were measured through FD-OCT RTVue. A study was done to determine the correlation between TM size, and other demographic and ocular parameters. Finally, the reproducibility of the measurements was assessed for a subgroup of 50 eyes from 50 patients.

REsults. We were able to measure the TM in $91.1 \%$ of the total eyes studied. The mean TM length was $496.99 \pm 92.77 \mu \mathrm{m}$ (range, 275-800), TM thickness was $174.16 \pm 28.14 \mu \mathrm{m}$ (range, 100-276), and TM area was $0.069 \pm 0.031 \mathrm{~mm}^{2}$ (range, 0.023-0.133). No differences were found in terms of length and area for sex, although the TM was slightly thicker in men $(P$ $=0.046)$. No correlation was observed between the TM measurements and any of the studied demographic or ocular parameters $(R<0.09, P<0.001)$. Intra- and interobserver reproducibility of the measurements were good (intraclass correlation coefficient $>0.750$, $P<0.001)$.

Conclusions. The FD-OCT is an effective and reproducible examination technique to measure the length, thickness, and area of the TM in vivo.

Keywords: trabecular meshwork, optical coherence tomography, glaucoma anterior segment
$T_{\mathrm{t}}^{\mathrm{h}}$ he iridocorneal angle has been assessed traditionally through gonioscopy, ${ }^{1,2}$ which allows for its direct visualization and, in particular, that of the trabecular meshwork (TM). The TM is an essential structure of the angle and is responsible for the drainage of aqueous humour. However, gonioscopy is a contact technique that presents certain disadvantages, such as subjectivity and a limited evaluation of the surface of the angle structures. That is the reason why it does not allow for the accurate measurement of the structures under study, among which is the TM.

To date, the leading imaging techniques for the assessment of the iridocorneal angle have been ultrasonic biomicroscopy $(\mathrm{UBM})^{3,4}$ and devices based on Scheimpflug technology. 5,6 However, the resolution of a Scheimpflug camera is inferior to that of optical coherence tomography (OCT). ${ }^{7}$ Besides, UBM is an invasive and time-consuming technique. ${ }^{3,4}$

In recent years, $\mathrm{OCT}^{8,9}$ has emerged as an alternative for assessing the iridocorneal angle..$^{10,11}$ The earlier time-domain OCT models allowed for the measurement of the angle width ${ }^{12}$ with the main objective of identifying those subjects with potentially occludable angles or those at risk of angle closure $^{13,14}$; however, they offered an image poor in detail. Optical coherence tomography has evolved from time-domain to Fourier domain (FD-OCT), or spectral-domain systems, and now offers enhanced signal-to-noise ratio, image acquisition speed, and resolution. With the current development of FDOCT, ${ }^{15,16}$ it is possible to give a completely new description of the angle, since it allows for the study of the various structures that make up the iridocorneal angle, as well as cross-sectional studies of the angle tissue. The earliest studies that identified the structures in the angle region ${ }^{17,18}$ were focused on the scleral spur and Schwalbe's line, ${ }^{19,20}$ because they were the visible structures, but they did not offer details on the TM. With the emergence of high-resolution FD-OCT or spectral domain anterior segment tomography, it now is possible to identify the TM swiftly and accurately as many have done. ${ }^{18,21-24}$

Interest in the study of the TM has increased in recent years, not only due to its relevance in the physiopathology of aqueous humour drainage in glaucoma, ${ }^{25}$ but also due to the emerging field of minimally invasive glaucoma surgery (MIGS) and the study of the drainage channels of the aqueous humour through the development of OCT. 
Most of the studies done up to the present, and especially those with a much higher number of participants, have been performed on Asian populations. ${ }^{26-28}$ Meanwhile, to our knowledge, no extensive studies on Caucasians are at our disposal.

The aim of this study was to measure the length, thickness, and area of the TM in vivo through FD-OCT in a healthy population to establish the standard of normality.

\section{MeTHODS}

A cross-sectional study was done on a sample of 1006 healthy subjects that were chosen consecutively among the patients who came for a medical check-up to the International Center of Advanced Ophthalmology in Madrid (Spain) between November 1, 2012 and June 30, 2013. These subjects first underwent a medical history and a complete eye examination. If they met the inclusion criteria and none of the exclusion, they all signed informed consent, and were explored with the OCT to complete the study. Therefore, all subjects selected were free of any ocular disease. The patients with iridotrabecular contact were excluded after the OCT scanning because it was a finding observed in this exploration. The study protocol adhered to the tenets of the Declaration of Helsinki. The Institutional Review Board of the center approved this study.

\section{Subjects}

Left eyes of each subject were randomly selected, adding up to a total of 1006 eyes. The inclusion criteria were aged between 18 and 85, and Caucasian. Subjects with previously diagnosed pathologies, or any ocular pathologies found in the exploration, such as glaucoma or a mature cataract, previous ocular surgery, including cataract and refractive surgery, a history of ocular trauma, and ocular or iridocorneal angle anomalies were excluded from the study. Those who presented physical or mental difficulties that could hinder examination and those who were treated with systemic medications that could modify the IOP also were excluded from the study.

\section{General Examination Protocol}

All patients underwent a standardized examination that included general medical history, measurement of visual acuity, slit-lamp anterior biomicroscopy, posterior segment ophthalmoscopy, tonometry with Canon TX 10 pneumotonometer (Canon, Inc., Tokyo, Japan), Pentacam (Oculus, Inc., Wetzlar, Germany) corneal topography, IOL Master (Carl Zeiss Meditec, Dublin, CA, USA) biometry, and OCT using Fourier Domain RTVue (Optovue, Inc., Fremont, CA, USA) on the same day. All examinations were performed in the afternoon to avoid diurnal variations (4-8 PM).

Examination with OCT RTVue, Pentacam, and IOL Master was performed in the same room with standardized mesopic lighting conditions, for which a SEKONIC Flashmate K-308S (SEKONIC, Tokyo, Japan) light meter was used at 7 EV or 320 lux.

Pentacam was used to measure the volume and depth of the anterior chamber (measured from the epithelium) and central corneal thickness (CCT). The IOL Master was used to measure the axial length, as well as the anterior chamber, and pupillary diameters.

\section{OCT Examination}

Examination was performed using an FD-OCT RTVue 100 (Optovue, Inc.), which has a scan speed of 26,000 A-scans per second, an 840-nm laser diode, and a 5- to 8- $\mu \mathrm{m}$ axial resolution. A CAM-L lens (Optovue, Inc., Fremont, CA, USA) was used for the analysis of the anterior segment. This lens is used to perform telecentric scans in which the OCT beams always is parallel to the main corneal axis.

Following our study protocol, examinations were performed with the pupil in mesopic conditions and with the device's software set to Angle mode. Analyses of the nasal and temporal sectors ( 3 and 9 o'clock) were performed. On this mode, a $3-\times 2.3-\mathrm{mm}$ area covered by 32 B-scans, each comprised of 1024 A-scans, centered on the limbus was modeled with 0.04 seconds in duration for each B-scan.

The images were obtained by a previously trained examiner (JFV) with the patient sitting and looking ahead into a fixed point inside the device. The quality of the images was determined by high Strength Signal Intensity (SSI), whereby images were chosen when this index was above 30 . Examination was performed three times in each sector, and the examiner chose the image that had the best quality and lowest noise.

The TM was identified as a hyperreflective tissue, as part of the interior of the scleral sulcus, which was visualized as a hyporeflective arc surrounding the TM or, as described by Kagemann et al., ${ }^{22}$ the TM interface shadow. The TM had a trapezoidal shape, demarcated on its anterior side by Schwalbe's line and on its posterior side by the scleral spur. It filled part of the inner third of the scleral thickness. The length, thickness, and area of the TM were measured directly using the software provided with the device. The length was measured from the hyperreflective point closest to the scleral spur to the hyperreflective point closest to Schwalbe's line. The thickness was measured at the halfway point of the TM length, and the area was measured by tracing the trapezoidal shape demarcated by the hyperreflective tissue. The surrounding hyporeflective arc or TM interface shadow was excluded from the measurement (Fig. 1).

Besides the TM measurements, OCT was used to measure conventional angle parameters, such as the anterior chamber angle (ACA), angle opening distance at $500 \mu \mathrm{m}$ to the scleral spur $\left(\mathrm{AOD}_{500}\right)$, iridotrabecular space area at $500 \mu \mathrm{m}$ to the scleral spur $\left(\mathrm{TISA}_{500}\right)$, and the iris thickness.

The ACA was measured by tracing a line between the angle recess towards the beginning of the TM and tracing the other line on the surface of the iris to the perpendicular point at the beginning of the TM. The $\mathrm{AOD}_{500}$ was calculated as the perpendicular distance measured from the TM at $500 \mu \mathrm{m}$ anterior to the scleral spur to the anterior iris surface. ${ }^{4}$ The TISA $_{500}$ was an area bounded anteriorly by the AOD, posteriorly by a line drawn from the scleral spur perpendicular to the plane of the inner scleral wall to the opposing iris, superiorly by the inner corneoscleral wall, and inferiorly by the iris surface. ${ }^{28}$ The iris thickness was measured at the perpendicular point $500 \mu \mathrm{m}$ to the scleral spur $\left(\mathrm{IT}_{500}\right)$.

\section{Reproducibility}

We chose 50 left eyes from 50 patients at random to assess the reproducibility of the TM measurements by OCT. In this subgroup the temporal and nasal sectors were measured to observe their correspondence. Left and right eyes of the 50 patients were included to assess left-right differences and their correlation.

Two observers (JFV and JGB) independently measured the TM images to assess interobserver reproducibility. One observer (JFV) repeated the scanning with OCT and the TM measurements two weeks after the first examination to determine intraobserver reproducibility. 

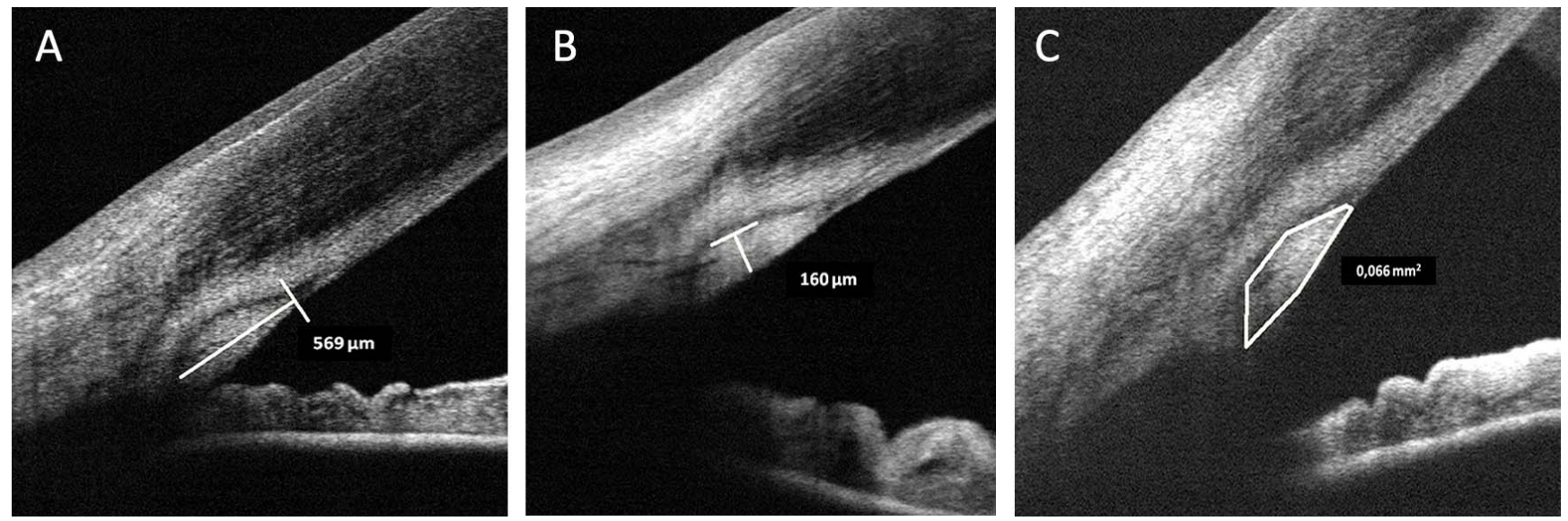

Figure 1. Example of TM measurements. (A) TM length. (B) TM thickness. (C) TM area.

\section{Statistical Analysis}

The statistical analysis was performed using SPSS (Statistical Package for Social Sciences, v18.0; SPSS, Inc., Chicago, IL, USA). Quantitative data were described as the mean and SD, while qualitative data were described as the frequency distribution. We applied the Kolmogorov-Smirnov test to assess the normal distribution of the measured variables. Partial correlation coefficients (adjusted for the other parameters studied) of length, thickness, and area of the TM were calculated. The intraclass correlation coefficient (ICC) was calculated for quantitative variables as a measure of interobserver and intraobserver reproducibility to assess the reliability of the FD-OCT measurements. Multivariate linear regression model was performed to adjust for possible intersex difference in the TM length, thickness, and area, with other parameters, such as anterior chamber depth (ACD), axial length, age, and refraction. The coefficients of variation (CV) also were calculated. The sample size was calculated observing that at least 582 patients had to be studied for the TM length, 116 for the TM thickness, and 796 for the TM area to detect a 10\% difference with $\alpha$ of 0.05 and $\beta$ of 0.10 . Statistical significance was set at $P<0.05$.

\section{RESULTS}

Iridotrabecular contact was observed in 17 eyes (1.6\%). These measurements were excluded because they could cause alterations to the TM, being finally studied in 989 eyes.

The mean age of patients was $49.09 \pm 15.21$ years (range, $18-84)$ with 615 women (61.14\%). The mean IOP was $15.85 \pm$ $3.49 \mathrm{~mm} \mathrm{Hg}$ (range, 6-25) and the mean spherical refractive error was $-0.40 \pm 3.55$ diopters (range, -14 to 8.25 ). The rest of the parameters studied are shown in Table 1.
We were able to measure the ACA in 939 eyes (94.9\%) in the temporal sector averaging at $35.80^{\circ} \pm 12.26^{\circ}$ (range, $1.50^{\circ}$ $76.11^{\circ}$ ). We could measure $\mathrm{AOD}_{500}$ and $\mathrm{TISA}_{500}$ in $90.8 \%$ of the total number of eyes. The mean $\mathrm{AOD}_{500}$ was $542.58 \pm 285.44$ $\mu \mathrm{m}$ (range, 15-1755) and the mean TISA 500 was $0.195 \pm 0.103$ $\mathrm{mm}^{2}$ (range, $0.02-0.62 \mathrm{~mm}^{2}$ ). The $\mathrm{IT}_{500}$ was $395.54 \pm 71.77$ $\mu \mathrm{m}$ (range, 194-910).

We identified and measured the TM in 901 eyes (91.1\%) in the temporal sector. The mean TM length was $496.99 \pm 92.77$ $\mu \mathrm{m}$ (range, 275-800), TM thickness was 174.16 $\pm 28.14 \mu \mathrm{m}$ (range, 100-276), and the TM area was $0.069 \pm 0.031 \mathrm{~mm}^{2}$ (range, $0.023-0.133 \mathrm{~mm}^{2}$ ). All three variables were normally distributed.

\section{Correlation Study}

There were no statistically significant differences in terms of length and area of the TM between male and female patients after adjustment with age, refraction, axial length, and ACD (difference $=5.12 \mu \mathrm{m}, P=0.376$ for the length; and difference $=0.002 \mathrm{~mm}^{2}, P=0.189$ for the area, respectively). However, TM was significantly thicker in male than in female patients. Men had an average TM $3.21 \mu \mathrm{m}$ thicker than women, which was statistically significant $(P=0.046)$.

Table 2 shows the partial correlation coefficients between TM size (length, thickness, and area) as measured in the temporal sector and the rest of the studied parameters. All of these correlations were $R<0.09$.

\section{Reproducibility}

Intraobserver reproducibility for the measurements of the TM was ICC $=0.826$ for TM thickness, which was higher than that for the area and length of the TM (ICC $=0.889$, Table 3). Interobserver reproducibility was $\mathrm{ICC}=0.750$ for $\mathrm{TM}$

Table 1. Values Obtained in the Population Studied by Scanning With Pentacam and IOL Master

\begin{tabular}{|c|c|c|c|c|c|c|}
\hline \multirow[b]{2}{*}{ Parameters } & \multicolumn{3}{|c|}{ Pentacam } & \multicolumn{3}{|c|}{ IOL Master, mm } \\
\hline & $\mathrm{ACD}, \mathrm{mm}$ & $\mathrm{CCT}, \mu \mathrm{m}$ & $\mathrm{ACV}, \mathrm{mm}^{\mathbf{3}}$ & Axial Length & AC Diameter & Pupillary Diameter \\
\hline Mean & 3.35 & 550.58 & 161.8 & 23.87 & 12.11 & 4.26 \\
\hline $\mathrm{SD}$ & 0.43 & 31.88 & 48.0 & 1.54 & 0.41 & 0.98 \\
\hline Maximum & 4.61 & 643 & 265 & 33.61 & 13.40 & 6.45 \\
\hline Minimum & 2.16 & 474 & 67 & 20.02 & 10.90 & 2.1 \\
\hline
\end{tabular}

$\mathrm{ACV}$, anterior chamber volume; AC diameter, anterior chamber diameter. 
TABLE 2. Partial Correlation Coefficients (Adjusted for the Other Parameters Studied) of Length, Thickness, and Area of the TM Measured in the Temporal Sector With Other Parameters Evaluated

\begin{tabular}{|c|c|c|c|c|c|c|}
\hline \multicolumn{7}{|c|}{ Partial Correlation Coefficients } \\
\hline Parameters & TM Length, $R$ & $P$ Value & TM Thickness, $R$ & $P$ Value & TM Area, $R$ & $P$ Value \\
\hline ACA & 0.007 & 0.035 & 0.001 & 0.044 & 0.041 & 0.042 \\
\hline ACD & 0.028 & $<0.001$ & 0.086 & $<0.001$ & 0.053 & 0.031 \\
\hline Axial length & -0.014 & 0.043 & -0.033 & 0.011 & -0.036 & 0.014 \\
\hline Spherical refractive error & -0.040 & $<0.001$ & -0.085 & $<0.001$ & -0.093 & $<0.001$ \\
\hline Age & 0.004 & 0.039 & 0.023 & 0.048 & 0.009 & 0.045 \\
\hline IOP & 0.031 & 0.200 & -0.054 & 0.027 & -0.013 & 0.594 \\
\hline CCT & -0.025 & 0.305 & 0.035 & 0.158 & -0.002 & 0.877 \\
\hline $\mathrm{ACV}$ & 0.055 & 0.025 & -0.005 & 0.013 & 0.033 & 0.007 \\
\hline Pupillary diameter & 0.002 & 0.530 & 0.005 & 0.640 & -0.019 & 0.451 \\
\hline AC diameter & -0.066 & 0.008 & -0.075 & 0.002 & -0.027 & 0.273 \\
\hline Iris thickness & 0.012 & 0.037 & 0.013 & 0.063 & 0.009 & 0.048 \\
\hline $\mathrm{AOD}_{500}$ & 0.001 & 0.065 & -0.069 & 0.005 & 0.012 & 0.031 \\
\hline TISA $_{500}$ & -0.058 & 0.017 & -0.008 & 0.231 & -0.049 & 0.046 \\
\hline
\end{tabular}

thickness, which was higher than that for the area and length of the TM (ICC $=0.831$, Table 4).

The average intrapatient right eye-left eye correlation was $R=0.325(P<0.001)$ for the length of the TM, with a right eye-left eye TM length variability equal to or higher than $20 \%$ in $38.2 \%$ of the patients. For the thickness the average intrapatient right eye-left eye correlation was $R=0.411(P<$ 0.0001 ) with a variability equal to or higher than $20 \%$ in $29.4 \%$ of the patients. For the area the average intrapatient right eyeleft eye correlation was $R=0.359(P<0.0001)$ with a right eye-left eye TM area variability equal to or higher than $20 \%$ in $52.6 \%$ of the patients.

The correlation between the temporal and nasal sector was $R=0.299$ for the TM length $(503.74 \pm 99.65$ vs. $498.32 \pm$ $93.10 \mu \mathrm{m}, P=0.002), R=0.279$ for the TM thickness $(173.93$ \pm 25.84 vs. $171.83 \pm 24.14 \mu \mathrm{m}, P=0.005)$, and $R=0.299$ for TM area $\left(0.067 \pm 0.017\right.$ vs. $\left.0.066 \pm 0.012 \mathrm{~mm}^{2}, P<0.006\right)$.

\section{Discussion}

Optical coherence tomography has emerged in recent years as an alternative and complementary method in the assessment of the iridocorneal angle. ${ }^{29}$ Numerous investigators, such as Perera et $a_{1}{ }^{30}$ and Qin et al., ${ }^{20}$ have compared the findings obtained through gonioscopy and OCT, obtaining good concordance for the detection of narrow angles. Radhakrishan et al. ${ }^{31}$ and Dada et al. ${ }^{32}$ equally obtained good concordance between UBM and OCT, although the OCT had a higher resolution. However, to our knowledge, in no studies through gonioscopy or UBM were researchers able to quantify the size of the TM.

Many investigators have identified the TM by OCT, ${ }^{18,21-24}$ but only some, such as Usui et al. ${ }^{23}$ or Tun et al., ${ }^{33}$ have offered measurements of this structure. In our study, we were able to identify and measure the TM in $91 \%$ of cases, while Quek et al. ${ }^{24}$ were able to identify the TM using OCT Cirrus in $34.4 \%$ of cases, increasing to $67.8 \%$ when using OCT iVue. Wong et al. ${ }^{18}$ identified the TM in $62.2 \%$ also with Cirrus. These differences probably are due to the enhanced image quality offered by the RTVue 100 device.

The mean TM length in our study, measured in the temporal sector through FD-OCT in vivo in a healthy Caucasian population was $496.99 \pm 92.77 \mu \mathrm{m}$, and it had a broad range from 275 to $800 \mu \mathrm{m}$ (Fig. 2). The angle parameters that have been standardized in recent years, such as AOD and TISA, measure the angle width by tracing a perpendicular line at 500 $\mu \mathrm{m}$ from the scleral spur and assume that the TM fills that space. However, as we can observe from our study, the TM has a broad range and, thus, we consider it would be more accurate to measure the angle width from the start of the TM, or as suggested by others, such as Cheung et al. ${ }^{19}$ and Qin et al., ${ }^{20}$ from Schwalbe's line.

One of the earliest published studies focusing on the analysis of the TM through OCT was that of Tun et al., ${ }^{33}$ which was performed using a swept-source device. The main limitation to this study was that the TM was demarcated indirectly, locating it in the space that remains between the scleral spur and Schwalbe's line. Therefore, measurements of the thickness or the area of the TM could not be taken due to the fact that they were estimating and not directly identifying the structure. In their results, the mean TM length was $779 \pm$ $98 \mu \mathrm{m}$ (range, 554-1054) for the horizontal sectors and for a significantly lower number of subjects than in our research. In our study, we took measurements not only of the length, but also of the thickness and area of the TM, since the RTVue device allowed us to directly visualize the TM. Thus, in this study we also suggested reconsidering a new point of

Table 3. Values Obtained in the Study of Intraobserver Reproducibility

\begin{tabular}{|c|c|c|c|c|c|}
\hline Intraobserver Reproducibility & Mean $\pm \mathrm{SD}, \mu \mathrm{m}$ & ICC & $\begin{array}{l}\text { 95\% Limits of } \\
\text { Agreement }\end{array}$ & $P$ Value & $\mathrm{CV}, \%$ \\
\hline TM length measurement 1 & $503.74 \pm 99.65$ & 0.968 & $0.952-0.978$ & $<0.0001$ & 19.54 \\
\hline TM length measurement 2 & $498.45 \pm 98.53$ & & & & \\
\hline TM thickness measurement 1 & $173.93 \pm 25.84$ & 0.826 & $0.752-0.879$ & $<0.0001$ & 14.07 \\
\hline TM thickness measurement 2 & $173.63 \pm 24.44$ & & & & \\
\hline TM area measurement 1 & $0.067 \pm 0.017$ & 0.889 & $0.836-0.922$ & $<0.0001$ & 24.34 \\
\hline TM area measurement 2 & $0.066 \pm 0.016$ & & & & \\
\hline
\end{tabular}


TABLE 4. Values Obtained in the Study of Interobserver Reproducibility

\begin{tabular}{|c|c|c|c|c|c|}
\hline Interobserver Reproducibility & Mean $\pm S D, \mu \mathrm{m}$ & ICC & $\begin{array}{l}\text { 95\% Limits of } \\
\text { Agreement }\end{array}$ & $P$ Value & $\mathbf{C V}, \%$ \\
\hline TM length observer 1 & $503.74 \pm 99.65$ & 0.933 & $0.902-0.954$ & $<0.0001$ & 19.56 \\
\hline TM length observer 2 & $497.12 \pm 96.26$ & & & & \\
\hline TM thickness observer 1 & $173.93 \pm 25.84$ & 0.750 & $0.650-0.824$ & $<0.0001$ & 14.86 \\
\hline TM thickness observer 2 & $177.76 \pm 26.97$ & & & & \\
\hline TM area observer 1 & $0.067 \pm 0.017$ & 0.831 & $0.758-0.883$ & $<0.0001$ & 25.46 \\
\hline TM area observer 2 & $0.065 \pm 0.020$ & & & & \\
\hline
\end{tabular}

reference for angle measurements since there still is TM beyond the $500 \mu \mathrm{m}$ standard.

Cheung et al. ${ }^{19}$ state that the mean TM length for the horizontal sectors in their study was $467 \pm 60 \mu \mathrm{m}$, while Usui et al. ${ }^{23}$ obtained a mean TM length of $466.9 \pm 60.7 \mu \mathrm{m}$. Usui et al. ${ }^{23}$ also found no differences between the measurements from the nasal and temporal sectors. These data are more in accordance with those observed in our study.

We also have been able to measure the TM thickness and area through FD-OCT in our study, with both measurements displaying a broad range (Fig. 3). We have not found any reference to OCT-based TM thickness measurements in the literature. Usui et al. ${ }^{23}$ published the first study with OCT that contributed information about the area of the TM. They obtained a mean TM area of $0.067 \pm 0.058 \mathrm{~mm}^{2}$, which is very similar to the results from our study $\left(0.069 \pm 0.031 \mathrm{~mm}^{2}\right)$.

Intra- and interobserver reproducibility of the measurements obtained through FD-OCT was very $\operatorname{good}^{34}$ for TM length and area (ICC >0.83), while that for TM thickness also was good yet slightly lower (ICC $>0.75$ ). The CV in the intraobserver study was $19.54 \%$ for TM length, $14.07 \%$ for TM thickness, and $24.34 \%$ for TM area due to the broad range of the measurements of these variables. The reproducibility of OCT in the assessment of the iridocorneal angle has been demonstrated by various investigators, ${ }^{35,36}$ among which we may highlight the study of Tan et al. ${ }^{37}$ to assess the reproducibility of the angle measurements for inexperienced examiners and which demonstrated that OCT is a more objective and less examiner-dependent technique than gonioscopy. With regard to OCT-based TM measurements, Tun et al. ${ }^{33}$ obtained good reproducibility.

All correlations between the measurements of the TM (length, thickness, and area) and each of the other studied parameters were $R<0.09$. Thus, there was either weak or no correlation or the correlation was very weak with any of the studied parameters, among which we can highlight age $(P<$
0.039), spherical refractive error $(P<0.001)$, ACD $(P<0.031)$, ACA $(P<0.042)$, and axial length $(P<0.043)$. Under our hypothesis, this could suggest that the TM is a structure independent from the rest of the aforementioned factors. These results coincided with those obtained by Tun et al., ${ }^{33}$ who did not find any correlations between TM length and any of the demographic factors, such as sex or age. In our study there were no statistically significant differences between length and area of the TM in terms of sex, although we found the TM was slightly thicker in men $(3.21 \mu \mathrm{m}, P=0.046)$. We do not consider this a clinically relevant difference under our hypothesis. They did not find any correlations between TM length and any of the angle parameters, such as AOD and TISA either, nor did they find any correlation between TM length of the left and right eyes.

Although weak, there was a slight correlation between left and right eyes for the TM measurements in our study (from $0.325-0.411, P<0.001)$. There also was a slight correlation between the temporal and nasal sectors $(0.270-0.299, P<$ 0.006). However, there were differences between the left and right eyes in the intrapatient study in terms of the size of the TM, whereby a TM length difference equal to or higher than $20 \%$ between the left and right eyes was observed in $38.2 \%$ of the patients.

In our study, we didn't observe a correlation between the TM size (length-thickness or area) with the IOP in healthy subjects, although these results were not statistically significant. We believe that is an interesting issue for future research to study the relation between the TM size and IOP in glaucomatous eyes because TM is believed to have an important role in the regulation of IOP. It is well known that increased flow resistance is responsible for the elevated IOP characteristic of glaucoma, but actually the cause of this resistance increase is not known. Recently, Overby et al. ${ }^{38}$ reported that, rather than being seen as a simple mechanical barrier to filtration, the endothelium of Schlemm's canal is seen
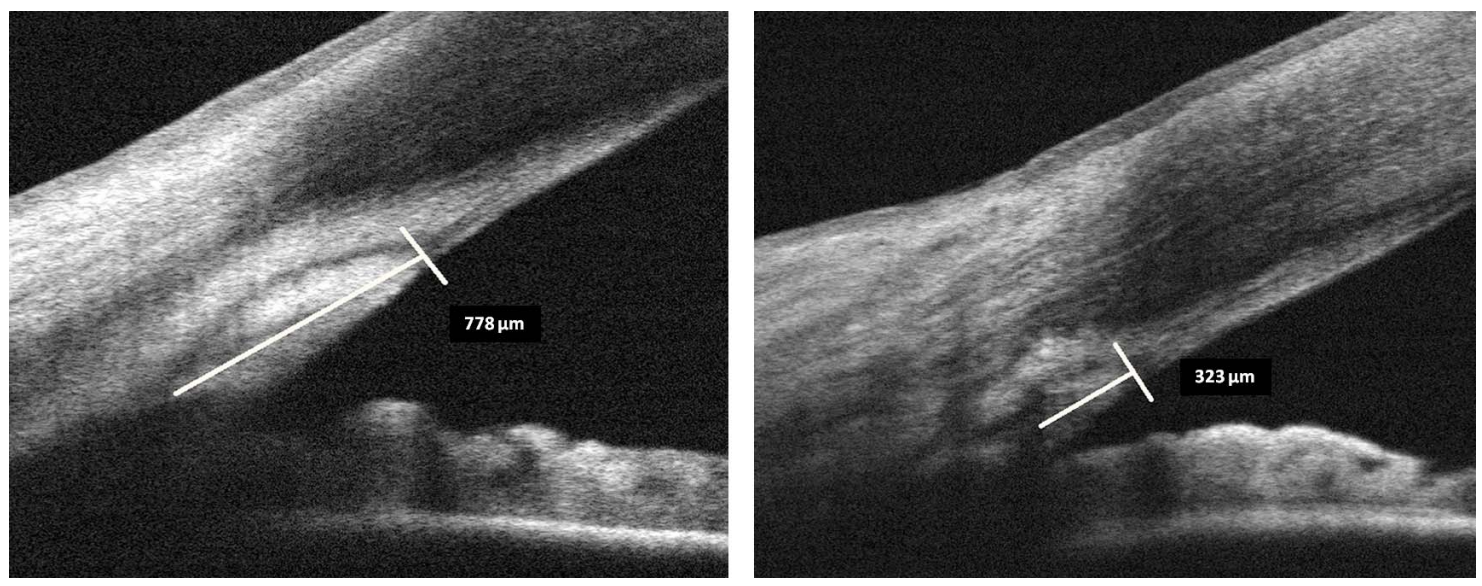

Figure 2. Measurements of the TM length. Left: Example of a long TM length. Right: Example of a short TM length. 

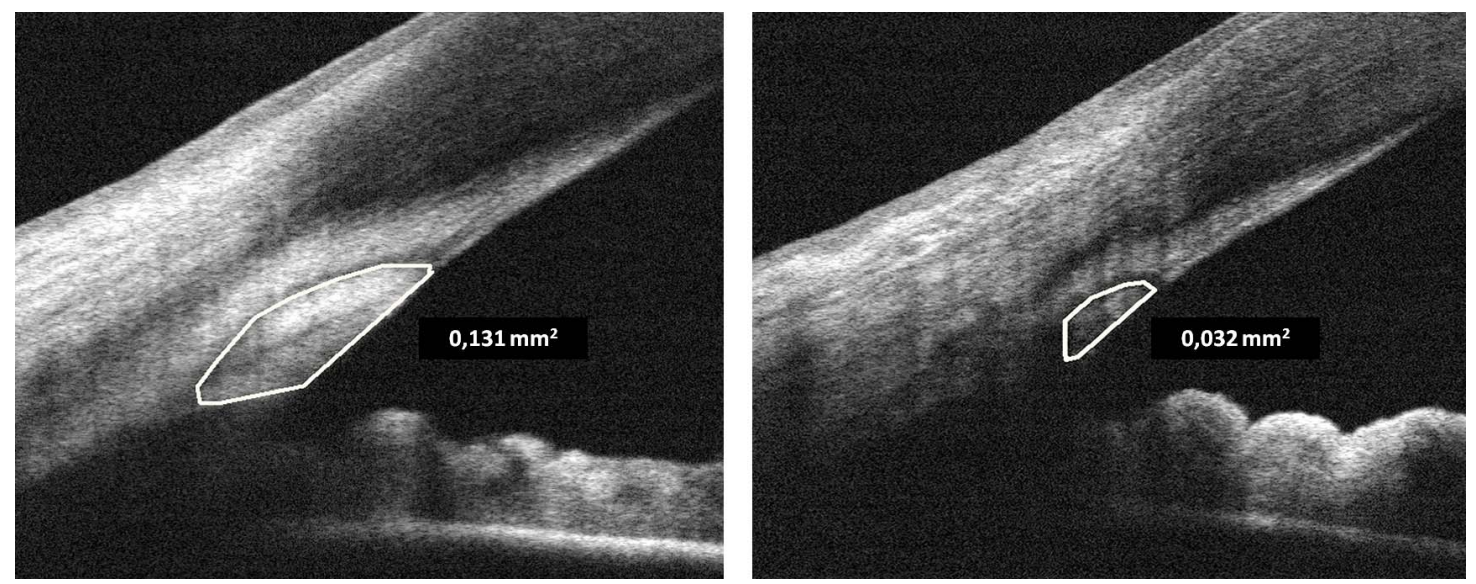

Figure 3. Measurements of the TM area. Left: Example of a large TM area. Right: Example of a small TM area.

instead as a dynamic material whose response to mechanical strain leads to pore formation and thereby modulates the resistance to aqueous humour outflow, and in the glaucomatous eye, this process becomes impaired.

Among the main limitations to our study is the fact that only one image from each sector was analyzed, although it is an average obtained from multiple scan samples of the same point. Only the horizontal sectors were assessed, due to the lower quality of the images obtained for the superior and inferior sectors and the need to manipulate the eyelids. With regard to the reproducibility study, examinations probably were not performed at the exact same spot and the measurements were taken manually without any kind of software-automatized correction.

This is the first study, to our knowledge, in which OCT has been used to characterize the TM and to measure its length, thickness, and area in vivo in a large Caucasian population of healthy subjects. The aim has been to contribute some reference values for the size of the TM to study glaucoma patients in the future and establish whether there is any variation in these measurements. We also will be able to understand the anatomical changes that occur in glaucoma surgery in more detail, especially in angle surgery. Finally, future studies may help us to better understand the physiopathology of glaucoma.

In conclusion, FD-OCT is a reliable technique to measure the length, thickness, and area of the TM in vivo in a healthy population.

\section{Acknowledgments}

The authors alone are responsible for the content and writing of the paper.

Disclosure: J.I. Fernández-Vigo, None; J. Garcia-Feijóo, None; J.M. Martínez-de-la-Casa, None; J. García-Bella, None; J.A. Fernández-Vigo, None

\section{References}

1. Shaffer RN, Schwartz A. Gonioscopy. Surv Ophthalmol. 1957; 2:389-409.

2. Spaeth GL. The normal development of the human anterior chamber angle: a new system of descriptive grading. Trans Ophthalmol Soc U K. 1971;91:709-739.

3. Pavlin CJ, Sherar MD, Foster FS. Subsurface ultrasound microscopic imaging of the intact eye. Ophthalmology. 1990;97:244-250.
4. Pavlin CJ, Harasiewicz K, Foster FS. Ultrasound biomicroscopy of anterior segment structures in normal and glaucomatous eyes. Am J Ophthalmol. 1992;113:381-389.

5. Lavanya R, Foster PJ, Sakata LM, et al. Screening for narrow angles in the Singapore population: evaluation of new noncontact screening methods. Ophthalmology. 2008;115: $1720-1727$.

6. Friedman DS, Gazzard G, Min CB, et al. Age and sex variation in angle findings among normal Chinese subjects: a comparison of UBM, Scheimpflug, and gonioscopic assessment of the anterior chamber angle. J Glaucoma. 2008;17:5-10.

7. Grewal DS, Brar GS, Jain R, Grewal SP. Comparison of Scheimpflug imaging and spectral domain anterior segment optical coherence tomography for detection of narrow anterior chamber angles. Eye (Lond). 2011;25:603-611.

8. Huang D, Swanson EA, Lin CP, et al. Optical coherence tomography. Science. 1991;254:1178-1181.

9. Izatt JA, Hee MR, Swanson EA, et al. Micrometer-scale resolution imaging of the anterior eye in vivo with optical coherence tomography. Arch Ophthalmol. 1994;112:15841589.

10. Wirbelauer C, Karandish A, Haberle H, Pham DT. Non contact goniometry with optical coherence tomography. Arch Ophthalmol. 2005;123:171-185.

11. Thomas R. Anterior segment optical coherence tomography. Ophthalmology. 2007;114:2362-2363.

12. Sakata LM, Wong TT, Wong HT, et al. Comparison of Visante and slit-lamp anterior segment optical coherence tomography in imaging the anterior chamber angle. Eye (Lond). 2010;24: 578-587.

13. Baek S, Sung KR, Sun JH, et al. A hierarchical cluster analysis of primary angle closure classification using anterior segment optical coherence tomography parameters. Invest Ophthalmol Vis Sci. 2013;54:848-853.

14. Nongpiur ME, Gong T, Lee HK, et al. Subgrouping of primary angle-closure suspects based on anterior segment optical coherence tomography parameters. Ophthalmology. 2013; 120:2525-2531.

15. Wylegala E, Teper S, Nowińska AK. Anterior segment imaging: Fourier-domain optical coherence tomography versus timedomain optical coherence tomography. J Cataract Refract Surg. 2009;35:1410-1414.

16. Asrani S, Sarunic M, Santiago C, Izatt J. Detailed visualization of the anterior segment using fourier-domain optical coherence tomography. Arch Ophthalmol. 2008;126:765-771.

17. Leung CK, Weinreb RN. Anterior chamber angle imaging with optical coherence tomography. Eye (Lond). 2011;25:261-267. 
18. Wong HT, Lim MC, Sakata LM, et al. High-definition optical coherence tomography imaging of the iridocorneal angle of the eye. Arch Ophthalmol. 2009;127:256-260.

19. Cheung CY, Zheng C, Ho CL. Novel anterior-chamber angle measurements by high-definition optical coherence tomography using the Schwalbe line as the landmark. $\mathrm{Br} J$ Ophthalmol. 2011;95:955-959.

20. Qin B, Francis BA, Li Y, et al. Anterior chamber angle measurements using Schwalbe's line with high resolution Fourier domain optical coherence tomography. J Glaucoma. 2013;22:684-688.

21. Bald M, Li Y, Huang D. Anterior chamber angle evaluation with fourier-domain optical coherence tomography. J Ophthalmol. 2012;2012:103704.

22. Kagemann L, Wollstein G, Ishikawa H, et al. Identification and assessment of Schlemm's canal by spectral-domain optical coherence tomography. Invest Ophthalmol Vis Sci. 2010;51: 4054-4059.

23. Usui $\mathrm{T}$, Tomidokoro A, Mishima $\mathrm{K}$, et al. Identification of Schlemm's canal and its surrounding tissues by anterior segment Fourier domain OCT. Invest Ophthalmol Vis Sci. 2011;52:6934-6939.

24. Quek DT, Narayanaswamy AK, Tun TA, et al. Comparison of two spectral domain optical coherence tomography devices for angle-closure assessment. Invest Ophthalmol Vis Sci. 2012; 53:5131-5136.

25. Quigley HA, Broman AT. The number of people with glaucoma worldwide in 2010 and 2020. Br J Ophthalmol. 2006;90:262267.

26. Nolan WP, See JL, Chew PT, et al. Detection of primary angle closure using anterior segment optical coherence tomography in Asian eyes. Ophthalmology. 2007;114:33-39.

27. Xu L, Cao WF, Wang YX, Chen CX, Jonas JB. Anterior chamber depth and chamber angle and their associations with ocular and general parameters: the Beijing Eye Study. Am J Ophthalmol. 2008;145:929-936.

28. Amerasinghe N, Foster PJ, Yin Wong T, et al. Variation of angle parameters in Asians: an anterior segment optical coherence tomography study in a Population of Singapore Malays. Invest Ophthalmol Vis Sci. 2009;50:2626-2631.

29. Smith SD, Singh K, Lin SC, et al. Evaluation of the anterior chamber angle in glaucoma: a report by the American Academy of Ophthalmology. Ophthalmology. 2013;120: 1985-1997.

30. Perera SA, Ho CL, Aung T, et al. Imaging of the iridocorneal angle with the RTVue spectral domain optical coherence tomography. Invest Ophthalmol Vis Sci. 2012;53:1710-1713.

31. Radhakrishnan S, Goldsmith J, Huang D, et al. Comparison of coherence tomography and ultrasound biomicroscopy for detection of narrow anterior chamber angles. Arch Ophthalmol. 2005;123:1053-1059.

32. Dada T, Sihota R, Gadia R, Aggarwal A, Mandal S, Gupta V. Comparison of anterior segment optical coherence tomography and ultrasound biomicroscopy for assessment of the anterior segment. J Cataract Refract Surg. 2007;33:837-840.

33. Tun TA, Baskaran M, Zheng C, et al. Assessment of trabecular meshwork width using swept source optical coherence tomography. Graefes Arch Clin Exp Ophthalmol. 2013;251: 1587-1592.

34. Altman DG. Statistics in medical journals: developments in the 1980s. Stat Med. 1991;10:1897-1913.

35. Radhakrishnan S, See J, Smith SD, et al. Reproducibility of anterior chamber angle measurements obtained with anterior segment optical coherence tomography. Invest Ophthalmol Vis Sci. 2007;48:3683-3688.

36. Liu S, Yu M, Ye C, Lam DS, Leung CK. Anterior chamber angle imaging with swept-source optical coherence tomography: an investigation on variability of angle measurement. Invest Ophthalmol Vis Sci. 2011;52:8598-8603.

37. Tan AN, Sauren LD, de Brabander J, et al. Reproducibility of anterior chamber angle measurements with anterior segment optical coherence tomography. Invest Ophthalmol Vis Sci. 2011;52:2095-2099.

38. Overby DR, Zhou EH, Vargas-Pinto R, et al. Altered mechanobiology of Schlemm's canal endothelial cells in glaucoma. Proc Natl Acad Sci U S A. 2014;111:13876-13881. 